\title{
La mediación de la Organización de la Unidad Africana durante los conflictos del Cuerno de África (1963-1991)
}

\section{The Organisation of African Unity's Mediating Role During the Conflicts in the Horn of Africa (1963-1991)}

\author{
PABLO ARCONADA-LEDESMA \\ Universidad de Valladolid, España
}

Resumen: Esta investigación tiene el objetivo de profundizar en el papel de la Organización de la Unidad Africana (OUA) en la resolución y la mediación de conflictos en la región del Cuerno de África durante el periodo de la Guerra Fría. Actualmente hay una controversia en torno a su actuación en estos procesos y es necesario examinar su actividad en la zona. De este modo, a través de un análisis de los documentos de la institución regional que hacen referencia a los conflictos de Ogaden (1964), la guerra de los Shifta (1963-1967) y la segunda guerra de Ogaden (1977-1978), se pretende arrojar un poco de luz sobre un tema específico que puede resultar muy novedoso. Para esta investigación se revisó la bi-

Recepción: 20 de octubre de 2020. / Aceptación: 12 de enero de 2021. 
bliografía de fuentes documentales de la OUA y la onU, así como de artículos de prensa.

Palabras clave: Organización de la Unidad Africana; Kenia; Etiopía; Somalia; conflictos.

Abstract: This research delves into the role of the Organisation of African Unity (OAU) in the resolution and mediation of conflicts in the Horn of Africa during the Cold War period. Its regional activities over the years in question warrant this analysis given the current controversy surrounding them. By studying this regional institution's records on the Ogaden conflict (1964), the Shifta War (1963-1967), and the second Ogaden War (1977-1978), the article sheds new light on a specific subject. The research is based on a review of OAU and UN documentary sources, as well as press articles.

Keywords: Organisation of African Unity; Kenya; Ethiopia; Somalia; conflicts.

\section{Introducción}

La Organización de la Unidad Africana (OUA) fue la principal entidad regional de carácter panafricano que buscaba la cohesión de los países recién descolonizados y continuar la lucha contra la colonización que aún persistía en el continente. A pesar de sus intenciones de mantener la paz y la estabilidad regional, lo cierto es que la OUA se encontró muy pronto con varios obstáculos en su labor.

Además de conflictos anteriores - como la secesión de Katanga el 11 de julio de 1960, apenas dos semanas después de que la República Democrática del Congo lograse la independencia (Kabunda y Ngoie 2010, 329)_, se generaron enfrentamientos en varias regiones de África, algunos incluso a los pocos meses del nacimiento de la OUA. Así, el 8 de octubre de 1963, Marruecos y Argelia iniciaron la Guerra de las Arenas por la disputa de los territorios de Hassi Beida y Tinyub (Torres García 2010, 155); en 1967, Biafra declaraba su independencia 
unilateral de Nigeria (Herteen y Dirk 2018, 3-4), sin olvidar las luchas internas de Sudán entre 1955-1972 (Poggo 2009, 1). Todos estos escenarios crearon un complejo rompecabezas en el que también influyeron los dos bandos de la Guerra Fría, que intentaron aumentar su influencia en el continente al reproducir el enfrentamiento global a escala regional.

Además, en el Cuerno de África se sucedieron guerras civiles y entre Estados cuyo principal epicentro fue Somalia. De este modo, entre 1963-1967 se desarrolló la conocida como guerra de los Shifta, un conflicto interno en Kenia que contó con el activo papel de Somalia; también se produjeron enfrentamientos interestatales: la primera guerra de Ogaden (1964), una disputa fronteriza, y la segunda guerra de Ogaden (1977-1978) entre Somalia y Etiopía (Arconada Ledesma, 2019). En esta región, la OUA se encontró de frente con un obstáculo colonial: el Territorio Francés de los Afars y los Isas que, hasta 1977, fue administrado por los galos y sería la última colonia al norte del ecuador (Arconada Ledesma 2020).

Ante estos acontecimientos, es oportuno profundizar en el papel que desempeñó la OUA durante los diferentes conflictos que tuvieron lugar en el Cuerno de África. Para desarrollar esta investigación, se realizó una revisión de la bibliografía concerniente al tema. Además, se utilizaron fuentes oficiales de la OUA, como resoluciones y decisiones de los diferentes órganos institucionales, así como actas de la ONU, discursos de los principales representantes políticos de países del Cuerno de África y artículos de prensa, todo lo cual ha permitido elaborar un detallado examen del rol desempeñado por la OUA en esta región.

\section{El papel de la ouA en el Cuerno de África}

Las investigaciones sobre la OUA y la resolución de conflictos ha sido un campo prolífico en el ámbito histórico, así como en el 
de las ciencias políticas en los últimos años. Las disputas fueron ciertamente frecuentes en las décadas siguientes a la descolonización, pero se produjeron según diferentes fórmulas: guerras entre Estados, enfrentamientos civiles, luchas subsidiarias... El principal debate, por tanto, ha girado en torno a la idea de la OUA como actor clave en los conflictos africanos en el periodo 1963-1991, esto es, durante la etapa de la Guerra Fría, aunque se advierte que, en relación con el estudio específico del Cuerno de África, sigue habiendo un hueco. Por ello es esencial examinar las medidas asumidas por la OUA en esta región.

Un claro ejemplo de obra centrada en el papel de la OUA fue The Unfinished Quest for Unity. Africa and the OAU, de 1973, en el que Cervenka analizó detalladamente la relación entre el continente y la institución. Este autor destacó algunas razones de por qué la OUA no tuvo la presencia ni el éxito esperado en este ámbito: los países africanos eran muy sensibles en cuanto a su soberanía interna; muchos de estos Estados se negaron a llevar sus disputas a un tribunal internacional, a no ser que tuvieran una situación legal favorable; consideraban que el derecho consuetudinario internacional era demasiado incierto, y que el derecho internacional era resultado de la práctica de Occidente que poco o nada tenía que ver con su recorrido y sus aspiraciones (Cervenka 1977, 49). Esta posición se debía al rechazo que suscitaba, entre los países africanos recientemente independizados, la cesión de su soberanía, teniendo en cuenta la trayectoria de dependencia política que habían sufrido como producto de la colonización. Ésta fue, sin duda, una de las grandes razones que incapacitaron a la OUA para intervenir activamente en los países miembros, en especial en aquellos que sufrían conflictos armados.

A raíz de este trabajo, diferentes investigadores retomaron el análisis del rol de la OUA en la mediación de conflictos regionales, pero con una postura contraria, al considerar que esta institución sí había tenido éxitos relativos. Así, Fredland (1973) examinó la situación del foro regional 10 años después de su fundación, y destacó el importante protagonismo de la 
OUA en la resolución de conflictos, como el de MarruecosArgelia o el de Etiopía-Somalia. Esta posición, con la entrada del nuevo siglo, fue apoyada con varias investigaciones, como la de Munyangwa y Vogt (2000), que indicaron que ya en la década de 1960 la resolución de conflictos por parte de la ouA había resultado ser de lo más exitosa en el Cuerno de África. Igualmente, trabajos posteriores han respaldado la tesis de la OUA como actor clave en el Cuerno de África, como señalaron Issa-Salwe (2001) o Fontrier $(2005,192)$, quien enfatizó el papel de otros Estados africanos como mediadores en los conflictos entre Etiopía y Somalia, pero bajo el amparo de la OUA, como fue el caso de Sudán en 1964. Estudios más actuales, como el de Edo y Olanrewaju (2012), han señalado también que la institución africana tuvo la capacidad de lograr la paz entre Etiopía y Somalia, y que estas decisiones se llevaron a buen puerto sin ningún tipo de intromisión internacional. Finalmente, Ahmed Mohammed $(2015,101)$ resaltó que los triunfos de la OUA en la década de 1960 se sumaron a los de la década siguiente, en especial en la segunda guerra de Ogaden.

Si bien todos estos investigadores conformaron una línea teórica basada en las buenas acciones de la OUA en la resolución de conflictos del Cuerno de África, pronto surgió otra visión, en la década de 1980. Esta línea exponía la incapacidad del foro regional de resolver las disputas. Una de esas primeras obras fue la de Bukarambe (1983), que apuntó al silencio de la OUA durante el conflicto de Ogaden, especialmente en relación con la intervención soviética y cubana. Por otro lado, Munya (1999) alabó los esfuerzos de la OUA y su Comité de Mediación para poner fin a los contenciosos, pero comentó que su desempeño resultó totalmente ineficaz en la región oriental. Hay obras más recientes, como la de Mengisteab (2014), con un estudio específico sobre la mediación de la ouA en el Cuerno de África en el que explica por qué su doctrina no evitó las guerras ni la desintegración de los países de la región. También Roberts (2014), aunque se centró en la guerra Tanzania-Uganda, destacó la inacción de la organización africana en África Oriental. 
No podemos tampoco dejar de mencionar a Belachew Yihun (2014), que evidenció la incapacidad de la OUA de intervenir en la guerra de Ogaden por su escasa autoridad decisoria sobre los Estados miembros.

De este modo, se observa cómo la producción científica se ha escindido en dos posiciones sobre el rol de la ouA en el periodo 1963-1991. No hay una tendencia teórica hegemónica, sino que las diferentes investigaciones arrojan datos contradictorios. Por ello, se consideró esencial una investigación específica del Cuerno de África que permita esclarecer en parte cuál fue la actuación de esa institución durante la Guerra Fría con una perspectiva histórica.

\section{El contencioso etíope-somalí (1964)}

La OUA se encontró, desde su nacimiento, en medio de un debate sobre las fronteras africanas. La mayor parte de los países independientes apoyó respetar las fronteras heredadas de la colonización con el fin de evitar la excesiva fragmentación territorial y soslayar posibles conflictos (Kasanda 2016, 188). Sin embargo, dos países se opusieron a estas tesis: Marruecos, cuya meta era construir un Gran Marruecos que abarcara la colonia del Río de Oro española y territorios de Argelia; y Somalia, que desde la unificación de 1960 tenía el objetivo expreso de unir a todos los pueblos somalíes que habitaban en la antigua Somalia italiana y la Somalilandia británica, Kenia, Etiopía y la Somalia francesa. Ambos Estados se encontraron con el muro de la OUA, que, en su Carta fundacional (OUA 1963), aprobó la inviolabilidad territorial y la sacralidad de las fronteras. De este modo, en el artículo 3o se comprometía a "respetar la soberanía e integridad territorial de cada Estado". El enfrentamiento fue visible desde la creación misma de la institución, de la que Marruecos se ausentó por su posición contraria al respeto de las fronteras (Rossi 1975). Además, las actas de la sesión fundacional revelaron la elevada tensión de 
los discursos entre Etiopía y Somalia. El presidente somalí, Aden Abullahi Osman, se pronunció de la siguiente manera: "Etiopía ha tomado posesión de una gran parte del territorio somalí sin consentimiento y en contra de los deseos de sus habitantes. El gobierno de Somalia no tiene intenciones de aumentar su territorio, está exigiendo que se aplique el derecho de autodeterminación" (Addis Ababa Summit 1963).

El primer ministro etíope, Aklilu Habte-Wold, respondió: "No hay ningún registro en la historia ni de un Estado ni de una nación somalí. Lamento decirlo, pero eso también es un hecho [...] Si no busca el engrandecimiento territorial, entonces, ¿qué es lo que busca? ¿En qué se basa para reclamar? ¿En un razonamiento lingüístico o en motivos religiosos?" (Addis Ababa Summit 1963).

El tono de estos discursos venía a demostrar la mala relación entre Somalia y Etiopía, que, un año después, dirimirían sus diferencias en el campo militar. Entre enero y marzo de 1964, se enfrentaron en una guerra fronteriza por el control de Ogaden. Aunque no está claro quién inició la agresión, lo cierto es que Etiopía, temiendo el avance somalí, decidió lanzar un ataque preventivo. Esta operación fue una respuesta al acercamiento a la URSS impulsado por Somalia con el objetivo de aumentar su capacidad militar. Como muestra un memorándum de la diplomacia estadounidense del 25 de octubre de 1963 (Brubeck 1963), ante la negativa de potencias occidentales como Estados Unidos, Italia y Gran Bretaña de ofrecer ese apoyo militar, el gobierno de Somalia podría optar por firmar un acuerdo de cooperación con la URSS, acuerdo que se materializó en noviembre de 1963 y que consistía en ayuda financiera por al menos 70 millones de dólares, cuya mitad estaría disponible de forma instantánea (Mealy 2012, 119). Esta ayuda se acompañó del envío de carros de combate, artillería, vehículos blindados, aviones y armas automáticas (Payton 1980, 500). Es evidente que este acuerdo generó gran temor en Etiopía ante un enemigo que podría iniciar un ataque en el corto plazo, y provocó una carrera armamentística y una 
reacción rápida de Addis Abeba para neutralizar cuanto antes una amenaza militar promocionada por Somalia.

Entre los planes de Addis Abeba estaba ganar la guerra y dictar las condiciones de paz de un futuro acuerdo. Las tropas etíopes iniciaron la ofensiva sobre puestos fronterizos y ciudades somalíes desde mediados de enero (Nkaisserry 1997, 10). The New York Times (1964a) se hizo eco del conflicto y registró el primer ataque el 17 de enero de 1964, en el que "tres aviones etíopes atacaron y destruyeron los controles de policía fronterizos de Gura Giome y El Maghet”. Durante los siguientes meses se desarrollaron combates a lo largo de toda la frontera, pero muy especialmente en el norte, cerca de Tuj Wajale, así como en las ciudades de Ferfer y Dollo, en el sur. También fueron comunes los bombardeos a ciudades somalíes más alejadas de la frontera, como Hargeisa (Markakis 2011, 147). Finalmente se firmó un alto el fuego el 31 de marzo, que se hizo efectivo el 8 de abril con la retirada de las tropas somalíes de Ogaden, hecho al que se refirió The New York Times (1964f) al día siguiente.

La derrota de Somalia reforzó también el reconocimiento internacional a Etiopía, que contaba con el apoyo de casi todos los países miembros de la oUA. Este respaldo conseguía ilegalizar cualquier aspiración de Somalia a modificar las fronteras. Durante la Primera Sesión Ordinaria de la OUA, en julio de 1964, celebrada en El Cairo, se aprobó la resolución AHG/ Res. 16 (I), en la que se especificó que la institución:

1. Reafirma solemnemente el estricto respeto por parte de todos los Estados miembros de la Organización de los principios establecidos en el párrafo 3 del artículo III de la Carta de la Organización de la Unidad Africana.

2. Declara solemnemente que todos los Estados miembros se comprometen a respetar las fronteras existentes en el logro de su independencia nacional (OUA 1964c).

Esta necesidad de reiterar la sacralidad de las fronteras africanas un año después de la fundación de la ouA responde 
al estallido de la guerra de Ogaden (1964) y de la guerra de los Shifta (1963-1967). Dicha situación también vino a demostrar la incapacidad de la organización de solucionar las disputas territoriales por la vía diplomática en su seno. Incluso unos meses antes, en un encuentro extraordinario en la ciudad de Lagos (Nigeria), el Consejo de Ministros aprobó la resolución CM/Res. 25 (II), en la que se publicaba un "Borrador del protocolo sobre mediación, conciliación y arbitraje” (OUA 1964f). Los conflictos que se habían producido entre Marruecos y Argelia, y los que se estaban desarrollando en el Cuerno de África, pusieron de relieve la emergente necesidad de crear un marco para la resolución de los enfrentamientos. El borrador se finalizó y aprobó durante la Conferencia de El Cairo, de manera que el nuevo protocolo se convertía en el marco para la resolución de controversias de cualquier tipo, al transformar los conflictos militares en disputas de tipo diplomático ("Organization of African Unity. Protocol of the Commission of Mediation, Conciliation and Arbitration" 1964).

En lo relativo a las controversias puntuales, la ouA se fue posicionando en cada una de ellas con la aprobación de diferentes resoluciones. Sobre las relaciones etíope-somalíes, aprobó la resolución ECM/Res. 3 (II) en febrero de 1964 en Dar es Salaam, sólo unas semanas después de que estallara el conflicto, y ahí llamaba

a los gobiernos de Etiopía y Somalia a que ordenen un inmediato cese del fuego y se abstengan de toda acción hostil. Recomienda a los gobiernos que adopten medidas para poner fin a todas las campañas de naturaleza provocativa o insultante. Pide a los gobiernos de Etiopía y de Somalia que actúen con el espíritu del párrafo 4 del artículo III de la Carta al entablar una negociación para la resolución pacífica de su disputa (OUA 1964a).

Es interesante la reacción de la organización, que, claramente, no se posicionó sobre el conflicto y trató de mediar entre los dos contendientes. Aunque la aspiración de Somalia pudo haber provocado el problema, lo cierto es que no se 
sabía quién lo había iniciado. Ante la situación, es lógico que la unión buscara un mecanismo para que los futuros enfrentamientos se solucionaran por la vía pacífica. No es por tanto una coincidencia que, en el siguiente encuentro de Lagos dos semanas más tarde, se creara el borrador del protocolo ya mencionado.

Este encuentro tuvo lugar entre el 24 y el 29 de febrero de 1964, y el Consejo de Ministros aprobó la resolución CM/Res. 16 (II), en la cual felicitaban a Etiopía y a Somalia por haber ordenado de inmediato un alto el fuego y haber establecido una atmósfera de distensión (ouA 1964d). Que esta resolución revelase que en febrero de 1964 los dos países habían abandonado la vía armada destaca la confusión de la guerra también en los medios de comunicación, que publicaron en febrero noticias de varios alto el fuego que nunca llegaron a cumplirse (Corriere della Sera 1964a, 1964b: 11 y 16 de febrero; The New York Times 1964b, 1964c y 1964d: 13 y 17 de febrero, 7 de marzo). Esto demuestra la falta de información veraz, así como la confusión que causaba el conflicto en la ouA y la escasa efectividad de las resoluciones de la institución africana en la práctica.

Si bien hay autores que defienden la tesis de que la paz se alcanzó gracias a los esfuerzos de la OUA, Fontrier (2005, 192193) indicó que la mediación de Sudán en el conflicto bajo los auspicios de la ouA había sido la principal razón de que la guerra etíope-somalí finalizara a principios de abril. Ambos Estados se reunieron desde el 24 de marzo en Jartum para buscar una salida pactada. El mismo punto de vista defiende Issa-Salwe (2001, 95-96), que además señaló el destacado papel que tuvo la OUA como foro para salir del trance, aunque esto se debió a que la ONU y su secretario general, U Thant, consideraron que aquel era el lugar de debate para un asunto interno africano. Evidentemente, Somalia prefería que el debate se internacionalizara porque podría conseguir más apoyos del exterior. En esa misma línea se encuentra la investigación de Edo y Olanrewaju $(2012,46)$, en la que se indica el éxito de la OUA en la resolución del conflicto territorial no sólo entre Somalia 
y Etiopía, sino también entre Marruecos y Argelia y en la guerra de Biafra, solución que se alcanzó sin ningún tipo de intromisión internacional.

¿Fue determinante el papel desempeñado por la OUA para el fin del conflicto? Aunque la labor de Sudán como mediador bajo los auspicios de la ouA fue importante, lo cierto es que su desempeño se ha exagerado. No hay que pasar por alto que la principal razón que llevó a Somalia a firmar el acuerdo de paz fue la incapacidad de hacer frente a las fuerzas armadas etíopes, que, hacia finales de marzo, tenían la situación casi bajo control. Además, Etiopía atacaba con frecuencia localidades somalíes con su aviación, lo que colocaba en una situación de debilidad a Somalia, tal como lo publicó Corriere della Sera (1964c) el 1 de marzo. Es cierto que Mogadiscio estaba involucrado de manera indirecta contra Kenia en la guerra de los Shifta, que más adelante analizaremos, lo que pudo acrecentar sus intenciones de cerrar cuanto antes uno de sus frentes bélicos. Por lo tanto, aunque la OUA fuera el marco en el que se puso fin a la guerra, no se puede afirmar que la resolución del conflicto se haya debido a los instrumentos de la OUA o a su presión, sino más bien a la superioridad etíope y a la búsqueda de un cese al fuego por parte de Somalia debido a su crítica situación. En ese sentido, coincido plenamente con las tesis de Mengisteab (2014), ya que el conflicto de Ogaden no se resolvió por la presión de la OUA, sino por la derrota militar de Somalia ante la enorme capacidad de Etiopía.

Finalmente, aunque el conflicto armado terminó, no se solucionó la pugna diplomática ni se encontró una fórmula para la paz definitiva en la disputa por Ogaden. Esto es visible en el memorándum 290 del Departamento de Estado de Estados Unidos publicado el 21 de marzo: "incluso una victoria total de Etiopía sobre Somalia, si es que eso fuera posible, no resolvería el problema de Somalia y además aumentaría la presión sobre el Imperio" (U.S. Department of State Office of the Historian 1999a). 


\section{La disputa entre Kenia y Somalia en el foro regional de la ouA (1963-1967)}

Paralelo al contencioso etíope-somalí, entre 1963-1967 se desarrolló un conflicto de tipo civil en el seno de Kenia, pero con una elevada implicación de Somalia. Desde principios de 1963, la población del Northern Frontier District (NFD), de mayoría somalí, inició una rebelión contra el gobierno de Jomo Kenyatta para reivindicar su independencia y su deseo de anexión a Somalia. De este modo, el periódico keniano The Daily Nation (1963) recogió, el 13 de noviembre, las palabras del primer ministro que señalaban a Somalia como el principal responsable de la situación en su país y exigió al gobierno somalí que dejara de reclamar el NFD como propio. Además, la preocupación de Kenia respecto a Somalia se debió al acuerdo de cooperación militar firmado con la URSS mencionado anteriormente y que alineaba a ambos países en dos bloques contrapuestos.

La guerra de los Shifta se desarrolló en el periodo de 19631967, y la implicación de Somalia se basó en el apoyo logístico y de armamento, al enviar sobre todo rifles coloniales británicos e italianos, ametralladoras tipo Brent y lanzagranadas (Mburu 2005, 134). Aunque las tropas somalíes nunca se internaron en el NFD, su sostén fue básico para mantener la lucha guerrillera encabezada por el Northern Frontier District Liberation Army (Turton 1972). Además, la radio nacional de Somalia, Radio Mogadishu, se utilizó como arma propagandística contra el gobierno de Kenia y fue clave para alentar a la población local a rebelarse. Así lo reflejan las actas del Parlamento de Kenia del 9 de noviembre de 1965: "Están alentando a la comunidad somalí a luchar con los shifta. Están diciendo que, si no luchas contra el gobierno de Kenia y el Parlamento de los kikuyus, no podrás volver a casa" (Asamblea Nacional de Kenia 1965, 366).

En cuanto al contencioso entre Kenia y Somalia, la OUA llegó a aprobar un total de tres resoluciones sobre la situación entre ambos países en el periodo 1963-1969. La institución afri- 
cana no tardó en pronunciarse sobre el conflicto indirecto del NFD, en el que se habían inmiscuido los gobiernos de Mogadiscio y Nairobi. De este modo, el 15 de febrero de 1964 la OUA pedía, a través de la resolución ECM/Res.4 (II), que los dos países tomaran las medidas necesarias para resolver la controversia y que se abstuvieran de seguir con acciones de provocación y propaganda mientras se buscaba una solución definitiva (OUA 1964b).

Unas semanas después, y tras ver que el conflicto seguía fuera de control, la OUA insistió, en el encuentro convocado en Lagos entre el 24 y el 29 de febrero de 1964, en que se retomara la negociación. Así, en el punto 2 de la resolución CM/Res. 17 (II) se invita "a los gobiernos de Kenia y Somalia a que abran lo antes posible negociaciones directas" (OUA 1964e). Lo que extraña en este punto es que, si Somalia no había entrado en guerra directa con Kenia, ¿por qué entonces la OUA señalaba directamente al Ejecutivo somalí y no lo trataba como conflicto civil? ¿Habría tenido algo que ver la situación en Ogaden? Es cierto que la decisión de llamar a Somalia a la negociación evidencia que había un acuerdo entre casi todos los Estados miembros de que el gobierno de Mogadiscio tenía algo que ver con el estallido de la guerra Shifta, hecho que puede demostrar que el conflicto de Kenia superaba ampliamente el marco de una guerra intraestatal. Quizá por haber sido dos fuerzas internas las que iniciaron el conflicto -los shifta en Kenia y los rebeldes del Western Somali Liberation Front en Ogaden (Mengo $2015,10)$ - , la OUA no fue capaz de hacer una diferenciación. No obstante, es evidente que hubo una divergencia esencial: en Ogaden se enfrentaron dos ejércitos regulares, pero esto no ocurrió nunca entre Kenia y Somalia (Whittaker 2015).

Llama la atención la resolución AHG/Res. 27 (II), aprobada en octubre de 1965, sobre "el problema de la subversión" (OuA 1965). Aunque en un principio da la sensación de que va dirigida a actores no africanos, hay dos puntos que podrían estar directamente relacionados con el conflicto del NFD, ya que, en primer lugar, se exigía: "abstenerse de realizar cualquier 
campaña de prensa o radio contra cualquier Estado miembro de la OUA". Es indudable que esta resolución hacía referencia a la guerra de comunicación que estaba impulsando el gobierno somalí con Radio Mogadishu. Igualmente, el punto 5 abogaba por "no crear disensiones dentro de los Estados miembros o entre ellos, que fomentaran o agravaran las diferencias raciales, religiosas, lingüísticas, étnicas o de otro tipo", algo que Somalia llevaba varios años incentivando en el NFD al defender la diferencia entre la mayoría de origen somalí y los musulmanes, frente a las poblaciones del resto de Kenia que eran, sobre todo, cristianos y de origen bantú (Mahmoud 2008).

Desde luego, la OUA intentó frenar la disputa regional, muestra de lo cual es la cantidad de resoluciones aprobadas, aunque ninguna de las partes se sentara a negociar hasta 1967. Precisamente, Rurumban (1965), en una publicación de The Daily Nation del 27 de marzo de 1965, recogió la intención del gobierno de Kenia de no frenar la escalada hasta lograr la total expulsión de los shifta del NFD. Muyangwa y Vogt (2000) enfatizaron la función diplomática de la OUA y argumentaron que en la década de 1960 la institución había logrado poner fin al contencioso en el NFD. De igual modo, Fredland $(1973,312)$ consideró que el foro regional tuvo éxito al proporcionar los medios para resolver varias controversias internas, aunque se debe considerar que los "medios" a los que se refería el autor respondían a la capacidad de generar espacios de negociación con apoyo en terceros países mediadores, como Tanzania y Zambia. Es evidente que la OUA no contó con mecanismos de corte intervencionista, debido a la limitación de participar directamente en los asuntos internos de los países miembros.

La última resolución aprobada por la OUA sobre las relaciones keniano-somalíes en la cumbre de Kinshasa del 11 al 14 de septiembre de 1967 anunció el restablecimiento de las negociaciones entre ambos países, las cuales se concretarían en un encuentro en Arusha un mes después. La resolución AHG/Res. 49 (IV) confirmaba la disposición de los dos eje- 
cutivos a reconocer la soberanía y la integridad territorial del otro, en lo que se convirtió en un claro primer paso para la normalización de las relaciones (OUA 1967). Además, en este documento se notificó la intención de ambos gobiernos de "abstenerse de realizar propaganda hostil a través de los medios de comunicación, como la radio y la prensa, contra el otro”, así como de "mantener la paz y la seguridad a ambos lados de la frontera y evitar cualquier tipo de pérdida de vida humana y material”. El 28 de octubre de 1967, los representantes de Somalia y Kenia se reunían en Arusha en presencia de Julius Nyerere y Keneth Kaunda para firmar un memorándum con el objetivo de reforzar la paz a ambos lados de la frontera. Este momento fue recogido en un reportaje de Reuters (1967) publicado el 30 de octubre.

Debemos señalar que la OUA, a pesar de que se esforzó por resolver el conflicto, no fue el móvil principal de la paz. La persistencia de la guerra, que se desarrolló durante cuatro años, evidenció la poca efectividad del organismo para imponerse y puso de relieve la falta de herramientas para frenarla. Como señaló Mukhtar (2003, 132), la institución africana intentó, entre 1965 y 1967, sentar a ambas partes en la mesa de negociación sin éxito. Aunque la paz que se firmó finalmente en octubre de 1967 tuvo lugar bajo los auspicios de presidentes nacionales como Keneth Kaunda de Zambia o Julius Nyerere de Tanzania, con el apoyo de la ouA, la principal razón de esta reconciliación se debió al cambio de gobierno interno de Somalia y su nueva política de détente con los Estados vecinos que impulsó el primer ministro Ibrahim Egal (Patman 1990, 88). Esta política coincidió con el alejamiento del nuevo gobierno de Somalia de la esfera de influencia de la URSS y el acercamiento a países occidentales, como Estados Unidos, Francia y Gran Bretaña, que eran también los principales aliados de Kenia. El 27 de julio de 1967, Ibrahim Egal envió un telegrama al Departamento de Estado de Estados Unidos con el fin de solicitar sus buenos oficios para lograr el entendimiento con varios países occidentales y estados vecinos (U.S. Department 
of State Office of the Historian 1999b). Este detalle es básico para comprender el nuevo alineamiento en el Cuerno de África que facilitó el acercamiento de Kenia y Etiopía con Somalia.

\section{La OuA y la segunda guerra de Ogaden}

El papel de la OUA en la resolución de conflictos durante su primera década fue bastante irregular, pero también demostró ser bastante activa con la aprobación de resoluciones y el impulso de negociaciones entre Estados. No obstante, en el periodo 1969-1991, el foro africano tuvo un papel menos relevante, quizá porque se hizo evidente que su capacidad de acción sobre los países miembros era bastante limitada y porque su principal objetivo - poner fin a la colonización- todavía no se había cumplido. No hay que olvidar que en la década de 1970 África austral seguía bajo dominio colonial. Angola y Mozambique eran colonias portuguesas, en Rodesia del Sur - actual Zimbabue- una minoría blanca se negó a iniciar el proceso de descolonización y declaró unilateralmente la independencia del Reino Unido en 1965. Igualmente, Sudáfrica y su protectorado de Namibia estaban bajo un régimen segregacionista, el apartheid (Chanaiwa 1993).

Con la revisión de la documentación se perciben tres periodos bien definidos sobre el papel de la OUA en relación con las disputas en el Cuerno de África en este periodo. En primer lugar, no hay resoluciones sobre los contenciosos de Somalia con Etiopía y Kenia entre 1969 y 1975, lo que tiene que ver con el entendimiento al que recurrió Siad Barre durante los primeros cinco años de su gobierno para consolidar su posición internamente (Lewis 2002, 220-226). Fue a partir de 1975 cuando el discurso de Somalia contra Etiopía se volvió mucho más agresivo e inició una guerra en junio de 1977 que se prolongó hasta marzo de 1978. Durante la primera fase del conflicto, y hasta noviembre de 1977, Somalia logró 
dominar casi todo Ogaden, si bien, a partir de ese mes, la intervención de algunos países comunistas, como la URSS, Cuba, Yemen del Sur y la República Democrática de Alemania (RDA), en apoyo a Etiopía, permitió a Addis Abeba vencer a las tropas somalíes y del Western Somali Liberation Front (Ayele 2014). En un artículo de The Ethiopian Herald (1978) se declararon, ya el 12 de febrero de 1978, las contundentes victorias de las fuerzas etíopes: "La valiente milicia popular y las fuerzas armadas regulares están dispensando gran valor y heroísmo en acciones militantes y están liquidando las fuerzas invasoras de Siad Barre".

La alineación de Etiopía con la URSS y Cuba potenció el acercamiento de Somalia a los aliados occidentales. Hasta el invierno de 1977, Somalia también se había asociado con la URSS, pero el estallido del conflicto en Ogaden y el apoyo expreso de Moscú a Etiopía empujó a Somalia a buscar nuevos aliados. A pesar de que Estados Unidos apoyó a Somalia a partir de febrero de 1978, no llegó a intervenir en el conflicto directamente, ya que éste finalizó en marzo de 1978, pero dio su apoyo logístico, financiero y militar posteriormente (Lockyer 2018).

Llama la atención que sólo se aprobase una resolución directamente relacionada con el conflicto de Ogaden y, además, que haya sido publicada en julio de 1978, esto es, cuando la guerra había finalizado. Podría pensarse que la OUA, además de centrar su objetivo en la descolonización, tenía como bandera no intervenir en los conflictos entre Estados, sobre todo por su limitada capacidad de acción. En cualquier caso, se analizarán aquí los dos temas de discusión en los que se centró la OUA durante las décadas de 1970-1980. Sobre su papel como intermediaria entre Etiopía y Somalia, la bibliografía ha arrojado visiones diferentes. Algunos expertos determinaron que esta institución tuvo un papel de liderazgo que consiguió enormes éxitos en lo que respecta a la guerra de Ogaden; otros, por el contrario, destacaron su incapacidad para poner fin a la escalada. En este apartado se tratará de dilucidar su posición respecto al conflicto fronterizo etíope-somalí en el periodo 1976-1980. 
La OUA sólo aprobó tres resoluciones respecto a la guerra que se vivía en el Cuerno de África y una se refería expresamente a Etiopía y a Somalia; las otras dos eran notas más generales que atañían a todo el continente. Durante la 14- Sesión Ordinaria de la Asamblea de Jefes de Estado de la ouA de 1977, se aprobó la resolución AHG/Res. 85 (XIV) sobre "la interferencia en los asuntos internos de los Estados africanos”. En este caso no se hace mención expresa de los dos países en conflicto, a pesar de que, a principios de julio de ese mismo año, era ya evidente la presencia de Somalia y sus guerrillas en territorio etíope. Aunque la resolución alude a un contexto general, no se pueden pasar por alto los puntos que relacionan esta resolución con el conflicto en Ogaden (OuA 1977).

En primer lugar, este documento recomendaba a todos los países africanos mantenerse "al margen de conflictos, especialmente aquellos de carácter ideológico, que emanan de fuera del continente". Es evidente la alusión al peligro que representaban para África las denominadas "guerras proxy" derivadas del conflicto de la Guerra Fría. En el caso concreto del Cuerno de África, más que un conflicto ideológico, lo que ocurrió fue una guerra fronteriza. No obstante, no hay que olvidar el apoyo que ambos Estados contendientes fueron recibiendo, sobre todo Etiopía y la colaboración soviética. Así, la OUA llamaba “a las fuerzas extranjeras, especialmente a las grandes potencias mundiales, a abstenerse de interferir en los asuntos internos africanos”. Más llamativo, sin duda, es el punto cuatro, en el que se expresaba que era necesario "prohibir el uso de sus territorios como base para actividades políticas subversivas contra otro Estado africano". Además del contencioso en Angola, esta denuncia encajaba perfectamente con la situación del Cuerno de África, ya que no se puede pasar por alto el apoyo de Somalia a grupos guerrilleros en Etiopía, como el Western Somali Liberation Front y el Northern Frontier District Liberation Front en Kenia.

Pero ¿por qué no se aprobó una resolución específica que señalara el contencioso etíope-somalí y a las potencias extran- 
jeras que estaban participando directa o indirectamente en el conflicto? A principios de julio de 1977 no estaba del todo claro cuál era la situación en Ogaden ni el nivel de participación de fuerzas foráneas en la región. En segundo lugar, era evidente que Etiopía contaba con el beneplácito de la URSS, y Somalia, en ese momento, no contaba con grandes apoyos extranjeros. Una resolución específica hubiera hecho que Somalia tomara esa decisión como un apoyo expreso de la OUA a su causa, a pesar de que había cometido una agresión contra otro Estado.

Hay otra resolución más, publicada en 1978, sobre el conflicto de Ogaden. En este caso, sí que es un documento que se refiere a la guerra entre Somalia y Etiopía. Sobre esa resolución, la OUA ordenaba "un cese inmediato de las hostilidades y de cualquier acto bélico de las dos partes interesadas: Etiopía y Somalia", y exigía "la retirada inmediata de las tropas de ambas partes de la frontera”. Además, pedía al presidente del Comité de Mediación de ese conflicto que aplicara todas las disposiciones "que le permitan asegurar la correcta y completa aplicación de esas medidas". Por último, retomaba las propuestas de la reunión ministerial de la OUA expuestas por la "Comisión de Buenos Oficios en la controversia entre Etiopía y Somalia”, celebrada en Jartum el 17 de julio de 1978, y en las que se decidió:

a) respetar las fronteras entre los Estados miembros de la OUA y la no injerencia en los asuntos internos de los Estados miembros; b) exigir a Etiopía y a Somalia que pusieran fin a todas las actividades militares y hostiles entre ellos; c) solicitar al órgano africano que prestara asistencia a los refugiados y que mediase para efectuar intercambio de prisioneros de guerra; d) continuar los esfuerzos de mediación con miras a encontrar una solución justa y duradera de la controversia (OUA 1978).

Tenemos que señalar que, de todas estas exigencias, ninguna se cumplió de manera inmediata. Además, aquellas que se solventaron años más tarde, no se debieron al papel de la OUA, sino al cambio de posiciones de Etiopía y Somalia, que 
relegaron el foro regional a un mero papel de observador. En primer lugar, la guerra no se resolvió plenamente hasta 1982, y no fue sino hasta 1988 cuando ambos Estados pusieron fin a una disputa que llevaba activa más de veinte años (Yagy 1990, 113). Tampoco la Comisión de Buenos Oficios de la OUA tuvo mucho que hacer frente a la guerra entre Etiopía y Somalia, que pasó de un conflicto interestatal a una disputa subversiva. De este modo, tanto Etiopía como Somalia siguieron interviniendo, a través de actores secundarios, en los asuntos de su Estado vecino; las actividades militares continuaron en activo, pues se enfrentaron sus ejércitos directamente al menos hasta 1982, tal como lo reflejan las actas de la 18a sesión plenaria de la Asamblea General de las Naciones Unidas (1982) del 5 de octubre de ese año.

Además, diversos argumentos completan el debate sobre el papel de la OUA en la resolución de conflictos en el Cuerno de África. En primer lugar, su incapacidad para mediar se debió a que ninguna de las partes enfrentadas tuvo ningún interés en resolver sus disputas por la vía diplomática. Así lo señaló Belachew Yihun $(2014,681)$ en su estudio sobre la guerra de Ogaden. El autor destaca que Somalia controlaba casi todo Ogaden en septiembre de 1977, lo que hizo que el régimen del Derg se negara a una negociación en la OUA. Era evidente que Etiopía no negociaría ninguna salida pacífica hasta que el ejército de Somalia fuera expulsado por completo del territorio etíope. Por su parte, Somalia se negaba a participar en cualquier negociación en la OUA, ya que ésta no reconocería la ocupación como legal, al ampararse en la sacralidad de las fronteras que expresaba su Carta Fundacional.

Como señaló Le Monde (22 de septiembre de 1977), el gobierno somalí esperaba que Etiopía reconociera el nuevo statu quo en Ogaden ante la presión de haber perdido todo el territorio. Si ambos Estados se negaban a participar en una mesa de negociación, el papel de la OUA sería inviable, ya que no había mecanismos que obligaran a los Estados a acatar sus órdenes. De esta forma, la Comisión de Buenos Oficios no 
tuvo capacidad para actuar. Al respecto, el investigador Munya $(1999,561)$ señaló que los esfuerzos de la OUA y su Comité de Mediación para resolver el conflicto fueron absolutamente ineficientes.

Otro hecho interesante y que restó importancia a la OUA en la resolución del conflicto fue la falta de denuncia de la intervención extranjera de la URSS y Cuba, algo que sí ocurrió en otra guerra contemporánea, en la que se avisó reiteradamente del apoyo militar y financiero a las partes enfrentadas: la guerra civil de Angola, como se observa en la resolución CM/Res. 474 (XXVII) de 1976 (ouA 1976). ¿A qué se debió esa diferencia? En primer lugar, en la guerra de Angola no había dos países enfrentados, sino varios bandos, por lo que denunciar a las potencias extranjeras no implicaba expresar apoyo a un Estado o a otro, lo que sí sucedía en el caso de la guerra en el Cuerno de África. Al respecto, Bukarambe $(1983,54)$ afirmaba que la OUA se negó “a condenar a los soviéticos-cubanos y su intervención contra Somalia porque el gobierno somalí lo hubiera considerado como una legitimación de la alteración de las fronteras por la fuerza y no por la vía pacífica”. Desde luego, el silencio de la OUA representó una falta de compromiso con el respeto a la soberanía estatal y a la no intervención en asuntos internos africanos. Esa medida demostró los límites de la institución a la hora de defender la integridad de los Estados africanos y frenar la influencia de la Guerra Fría en el continente.

A pesar de ello, hay autores que siguen considerando muy positivo el papel mediador de la OUA. Es el caso de Ahmed Mohammed $(2015,101)$ cuando afirma que el organismo fue clave en la resolución de los conflictos que se sumaron a los triunfos de la década de 1960. No concuerdo con esa visión, ya que no sólo en el caso de Ogaden, sino también en el de la guerra Uganda-Tanzania, que tuvo lugar en 1978, la participación de la ouA fue escasa, como ya señaló Roberts $(2014,697)$ en su investigación sobre este conflicto. 


\section{A modo de conclusión}

Es evidente que no hay consenso sobre el papel de la OUA en la mediación de conflictos en el continente africano durante el periodo de la Guerra Fría. Algunos autores siguen defendiendo que fue la principal protagonista, mientras que otros han destacado su limitada capacidad y el hecho de que la mayoría de esas disputas acabaron por razones internas, totalmente ajenas al foro africano.

Esta investigación demuestra que sigue siendo importante profundizar en el ámbito de la resolución de conflictos en el Cuerno de África para comprender mejor las causas que desembocaron en el fin de los contenciosos y entender el rol de la institución africana. Desde luego, no se niega aquí que la OUA demostró ser un foro muy activo en África Oriental, en especial durante su primera década. El elevado número de resoluciones y decisiones, así como la constitución del Comité de Mediación, Conciliación y Arbitraje, evidencian el interés del órgano regional en la resolución de conflictos. También se debe valorar que los países mediadores, como el caso de Sudán en 1964 o el de Zambia y Tanzania en 1967, lograron sentar en la mesa de negociación a Somalia tanto con Etiopía como con Kenia bajo los auspicios de la OUA. Quizás ésa fuera la mayor contribución de la OUA a la paz en el Cuerno de África: su capacidad de mediación entre las partes. Sin embargo, el papel de la organización africana fue limitado: no contaba con mecanismos específicos para frenar los problemas con un carácter intervencionista y los propios Estados africanos recelaban de cualquier intromisión en sus asuntos.

Por ello, no hay que olvidar que los conflictos en Ogaden y en el Northern Frontier District finalizaron por decisión interna de una o de todas las partes involucradas en la disputa, que hicieron muchas veces caso omiso de las decisiones de la OUA. Esto fue evidente en la primera guerra de Ogaden (1964), cuando la superioridad etíope obligó a Somalia a retirarse y a firmar el acuerdo de paz; o en la segunda guerra de Ogaden (1977-1978), 
en que la expulsión de Somalia de la región etíope, con apoyo de la URSS y Cuba, forzó al gobierno somalí a retirar sus tropas del país vecino y a firmar un alto el fuego. El caso de la guerra de los Shifta es algo diferente, ya que ambas partes, ante la incapacidad de lograr un triunfo en el campo militar, decidieron negociar una salida pacífica que se concretó en un encuentro de la OUA en Kinshasa en septiembre de 1967. Este acuerdo se alcanzó gracias a un cambio de gobierno interno en Somalia, donde el nuevo gabinete, encabezado por Ibrahim Egal, apostó por la paz con los Estados vecinos para poner fin al ciclo de violencia armada en el que su país se había visto inmerso.

Como conclusión, es posible determinar que la OUA desarrolló una participación muy activa durante su primera década de actividad al tratar de mediar entre los países en conflicto, aunque sus exigencias pocas veces fueron escuchadas. Su intervención fue mucho menos enérgica a partir de 1975, tal como se observó en el conflicto de Ogaden, en el que el organismo tan sólo aprobó una resolución que vio la luz meses después de que la agresión somalí sobre Ogaden hubiera terminado. Las escasas herramientas de intervención del ente regional, así como el recelo de los países miembros a que su soberanía fuera fiscalizada, explican por qué la OUA no tuvo una función determinante en el fin de los conflictos regionales del Cuerno de África.

\section{Referencias}

Addis Ababa Summit. 1963. Addis Ababa: Publications and Foreign Languages Press Department.

Ahmed Mohammed, Seid. 2015. "Organization of African Unity and the African Diplomacy". Izmir Review of Social Sciences 3(1): 95-108.

Arconada Ledesma, Pablo. 2019. "Guerras fronterizas en el Cuerno de África: el conflicto etíope-somalí y sus consecuencias”. En Del siglo XIX al XXI. Tendencias y debates: XIV Congreso de la Asociación de Historia Contemporánea, Universidad de Alicante 20-22 de 
septiembre de 2018, coordinado por Mónica Moreno Seco y editado por Rafael Fernández Sirvent y Rosa Ana Gutiérrez Lloret, 1853-1868. Alicante: Biblioteca Virtual Miguel de Cervantes. http://www.cervantesvirtual.com/nd/ark:/59851/bmc0947482 Arconada Ledesma, Pablo. 2020. "El proceso de descolonización de Yibuti: entre la influencia de Francia y la disputa etíope-somalí”. En África, un continente en transformación. Enfoques interdisciplinares, editado por César García Andrés, Jara Cuadrado Bolaños y Pablo Arconada Ledesma, 87-103. Valladolid: Universidad de Valladolid.

Asamblea General de las Naciones Unidas. 1982. 18a sesión plenaria, 37o periodo de sesiones, Nueva York, 5 de octubre de 1982. https://digitallibrary.un.org/record/36740?!n = es\# record-filescollapse-header

Asamblea Nacional de Kenia. 1965. Actas del Parlamento, tercera sesión, 2 de noviembre-10 de diciembre de 1965. https://books.

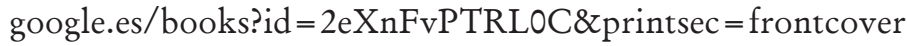
$\& \mathrm{hl}=$ es\&source $=$ gbs_ge_summary_r\&cad $=0 \# \mathrm{v}=$ onepage $\& \mathrm{q}$ $\& \mathrm{f}=$ false

Ayele, Fantahun. 2014. The Ethiopian Army: From Victory to Collapse, 1977-1991. Evanston: Northwestern University Press.

Belachew Yinun, Belete. 2014. "Ethiopian Foreign Policy and the Ogaden War: The Shift from 'Containment' to 'Destabilization', 1977-1991”. Journal of Eastern African Studies 8(4): 677-691. https://doi.org/10.1080/17531055.2014.947469

BRUBECK, William H. 1963. "Memorandum for the President [John F. Kennedy]”, 25 de octubre de 1963. Papers of John F. Kennedy. Presidential Papers. President's Office Files. Countries. Somali Republic: General, 1962-1963. https://www.jfklibrary.org/assetviewer/archives/JFKPOF/124/JFKPOF-124-004

Bukarambe, Bukar. 1983. "The Role and Impact of the OAU in the Management of African Conflicts". Survival: Global Politics and Strategy 25(2): 50-58. https://doi.org/10.1080/ 00396338308442084

CervenKa, Zdenek. 1977. The Unfinished Quest for Unity. Africa and the OAU. Londres: Friedmann.

Chanaiwa, David. 1993. "Southern Africa since 1935”. En General History of Africa. Vol. 8: Africa since 1935, editado por Ali A. Mazrui y Christophe Wondji, 249-284. París: UNESCO. 
Corriere della Sera. 1964a. "Mediazione del Sudan fra Etiopia e Somalia". 11 de febrero de 1964, p. 16.

Corriere della Sera. 1964b. "Somalia e Etiopia sospendono i combattimenti". 16 de febrero de 1964, p. 7.

Corriere della Sera. 1964c. "Fallita l'operazione Ogaden". 1 de marzo de 1964, p. 4.

EDO, Victor Osaro y Michael Abiodun Olanrewaju. 2012. “An Assessment of the Transformation of the Organization of African Unity (OAU) to the African Union (AU), 1963-2007". Journal of the Historical Society of Nigeria 21: 41-69. http://www.jstor.org/ stable/41857189

FonTrier, Marc. 2005. "La crise de 1963-1964 en Ogadén”. Annales d'Éthiopie 20: 181-194. https://doi.org/10.3406/ethio.2004.1074

FREDLAND, Richard. 1973. "The OAU after Ten Years: Can It Survive?”. African Affairs 72(288): 309-316. https://doi.org/10.1093/ oxfordjournals.afraf.a096388

Heerten, Lasse y A. Dirk Moses. 2018. "The Nigeria-Biafra War Postcolonial Conflict and the Question of Genocide”. En Postcolonial Conflict and the Question of Genocide. The Nigeria-Biafra War, 1967-1970, editado por A. Dirk Moses A. y Lasse Heerten, 169-203. Nueva York: Routledge.

Issa-SALWe, Abdisalam M. 2001. Cold War Fallout: Boundary Politics and Conflict in the Horn of Africa. Londres: Haan.

Kabunda BADI, Mbuyi y Germain Ngoie Tshibambe. 2010. "La secesión de Katanga revisitada o las incertidumbres de la creación del Estado-nación en el África postcolonial”. En Secesionismo en África, editado por Jordi Tomás, 329-365. Barcelona: Edicions Bellaterra.

KaSANDA, Albert. 2016. “Exploring Pan-Africanism's Theories: From Race-Based Solidarity to Political Unity and Beyond". Journal of African Cultural Studies 28(2): 179-195. https://doi.org/10.1 080/13696815.2016.1158093

Le Monde. 1977. "Le Front de Libération Somali est prêt à négocier avec l'Éthiopie”. 22 de septiembre de 1977. https://www. lemonde.fr/archives/article/1977/09/22/le-front-de-liberationsomali-est-pret-anegocier-avec-l-ethiopie_2869343_1819218. html

LEWIS, Ioan Myrddin. 2002. A Modern History of the Somali. Nation and State in the Horn of Africa. Oxford: James Currey. 
LOCKYER, Adam. 2018. “Opposing Foreign Intervention's Impact on the Warfare in Civil Wars: The Case of the Ethiopian-Ogaden Civil War, 1976-1980”. African Security 11(2): 181-199. https:// doi.org/10.1080/19392206.2018.1480141

Mahmoud, Hussein Abdullahi. 2008. "Seeking Citizenship on the Border: Kenya Somalis, the Uncertainty of Belonging, and Public Sphere Interactions". Ponencia presentada en la Codesria 12th General Assembly, Yaoundé, Camerún, 7-12 de diciembre de 2008.

Markakis, John. 2011. Ethiopia: The Last Two Frontiers. Rochester, NY: James Currey.

MBURu, Nene. 2005. Bandits on the Border: The Last Frontier in the Search for Somali Unity. Trenton, NJ: The Red Sea Press.

Mealy, Sam. 2012. "State-building in the Horn of Africa. The panSomali project and Cold War Politics during 1960". History Studies 13: 114-132. historystudies_13_mealy_state-building. pdf (ul.ie)

Mengisteab, Kidane. 2014. "The OAU doctrine on Colonial Boundaries and Conflicts of Separation in the Horn of Africa". En Self-Determination and Secession in Africa: The Post-Colonial State, editado por Redie Bereketeab, 38-50. Londres: Routledge.

Mengo, Francesco Maria. 2015. "Il Derg, Siad Barre e l'Ogaden. Due diverse concezioni di dittatura militare e il loro scontro in una guerra per procura". Diacronie. Studi di Storia Contemporanea 24(4): 1-17. http://www.studistorici.com/2015/12/29/ mengo_numero_24/

Mukhtar, Mohamed Haji. 2003. Historical Dictionary of Somalia. Lanham: Scarecrow Press.

Munya, Mweti. 1999. “The Organization of African Unity and Its Role in Regional Conflict Resolution and Dispute Settlement: A Critical Evaluation”. Boston College Third World Law Journal 19: 537-592.

Muyangwa, Monde y Margaret A. Vogt. 2000. An Assessment of the OAU Mechanism for Conflict Prevention, Management, and Resolution, 1993-2000. Nueva York: International Peace Institute. https://www.ipinst.org/2000/07/an-assessment-ofthe-oau-mechanism-for-conflict-prevention-management-andresolution-1993-2000 
Nkaisserry, Joseph K. 1997. The Ogaden War: An Analysis of its Causes and its Impact on Regional Peace on the Horn of Africa. Carlisle: U.S. Army War College. http://www.dtic.mil/dtic/ tr/fulltext/u2/a326941.pdf

"Organization of African Unity. Protocol of the Commission of Mediation, Conciliation and Arbitration”. 1964. International Legal Materials 3(6): 1116-1124. http://www.jstor.org/stable/20689845 OUA (Organización de la Unidad Africana). 1963. "Carta fundacional de la Organización de la Unidad Africana”, 25 de mayo de 1963. https://au.int/sites/default/files/treaties/7759-file-oau_charter_1963.pdf

ouA (Organización de la Unidad Africana). 1964a. ECM/Res. 3 (II), Second Extra-Ordinary Session of the Council of Ministers, Dar es Salaam, Tanzania, 12-15 de febrero de 1964. https://au.int/en/ decisions/council-ministers-meeting-its-second-extraordinarysession

OUA (Organización de la Unidad Africana). 1964b. ECM/Res. 4 (II), Second Extra-Ordinary Session of the Council of Ministers, Dar es Salaam, Tanzania, 12-15 de febrero de 1964. https://au.int/en/ decisions/council-ministers-meeting-its-second-extraordinarysession

ouA (Organización de la Unidad Africana). 1964c. "Border Disputes among African States”, AHG/Res. 16 (I), First Ordinary Session of the Assembly of Heads of State and Government, El Cairo, RAU, 17-21 de julio de 1964. https://au.int/en/ decisions/assembly-heads-state-and-government-first-ordinarysession-0

ouA (Organización de la Unidad Africana). 1964d. "Border Dispute between Ethiopia and Somalia”, CM/Res. 16 (II), Second Ordinary Session of the Council of Ministers, Lagos, Nigeria, 24-29 de febrero de 1964. https://au.int/en/decisions/council-ministersmeeting-its-second-extraordinary-session-0

OUA (Organización de la Unidad Africana). 1964e. "Dispute between Somalia and Kenya”, CM/Res. 17 (II), Second Ordinary Session of the Council of Ministers, Lagos, Nigeria, 24-29 de febrero de 1964. https://au.int/en/decisions/council-ministers-meeting-itssecond-extraordinary-session-0

OUA (Organización de la Unidad Africana). 1964f. "Draft Protocol on Mediation, Conciliation and Arbitration", CM/Res. 25 
(II), Second Ordinary Session of the Council of Ministers, Lagos, Nigeria, 24-29 de febrero de 1964. https://au.int/en/ decisions/council-ministers-meeting-its-second-extraordinarysession-0

OUA (Organización de la Unidad Africana). 1965. "Declaration on the Problem of Subversion”, AHG/Res. 27 (II), Second Ordinary Session of the Assembly of Heads of State and Government, Acra, Ghana, 21-26 de octubre de 1965. https://au.int/en/ decisions-129

OUA (Organización de la Unidad Africana). 1967. "Resolution on Mercenaries”, AHG/Res. 49 (IV), Fourth Ordinary Session of the Assembly of Heads of State and Government, Kinsasha, Congo, 11-14 de septiembre de 1967. https://au.int/sites/default/ files/decisions/9516-assembly_en_11_14_september_1967_assembly_heads_state_government_fourth_ordinary_session.pdf OUA (Organización de la Unidad Africana). 1976. "Resolution on Angola”, CM/Res. 474 (XXVII), Twenty-seventh Ordinary Session of the Council of Ministers, Port-Louis, Mauricio, 24 de junio-3 de julio de 1976. https://au.int/sites/default/files/ decisions/9595-council_en_24_june_3_july_1976_council_ministers_twenty_seventh_ordinary_session.pdf

OUA (Organización de la Unidad Africana). 1977. "Resolution on the Interference of the Internal Affairs of African States", AHG/ Res. 85 (XIV), Fourteenth Ordinary Session of the Assembly of Heads of State and Government, Libreville, Gabon, 2-5 de julio de 1977. https://au.int/sites/default/files/decisions/9524assembly_en_2_5_july_1977_assembly_heads_state_government_fourteenth_ordinary_session.pdf

OUA (Organización de la Unidad Africana). 1978. "Resolution on the Somalia/Ethiopia Conflict”, AHG/Res. 90 (XV), Fifteenth Ordinary Session of the Assembly of Heads of State and Government, Jartum, Sudán, 18-22 de julio de 1978. https://au.int/sites/default/ files/decisions/9525-assembly_en_18_22_july_1978_assembly_heads_state_government_fifteenth_ordinary_session.pdf Patman, Robert G. 1990. The Soviet Union in the Horn of Africa. The Diplomacy of Intervention and Disengagement. Cambridge: Cambridge University Press.

Payton, Gary D. 1980. “The Somali Coup of 1969: The Case for Soviet Complicity". The Journal of Modern African 
Studies 18(3): 493-508. https://doi.org/10.1017/ S0022278X00011629

Poggo, Scopas. 2009. The First Sudanese Civil War. Africans, Arabs, and Israelis in the Southern Sudan, 1955-1972. Nueva York: Palgrave Macmillan. https://doi.org/10.1057/ 9780230617988

Reuters. 1967. "Tanzania: Kenya and Somalia sign 'Memorandum of Understanding' on Border Dispute”. Arusha, Tanzania, 30 de octubre de 1967. Video. Reuters ID: LVAE7P1HGGSI09MNQ5JYD6FDBFF2. Screenocean.com, https://reuters.screenocean. $\mathrm{com} / \mathrm{record} / 293856$

ROBERTS, George. 2014. "The Uganda-Tanzania War, the Fall of Idi Amin, and the Failure of African Diplomacy, 1978-1979". Journal of Eastern African Studies 8(4): 692-709. https://doi.org/10.1080 /17531055.2014.946236

Rossi, Gianluigi. 1975. "The OAU: Result of a Decade". International Journal of Politics 4(4): 15-34. https://www.jstor.org/ stable/27868811

Rurumban, P. L. 1965. "We Won't give Inch of N.E.R.-Mungai”. The Daily Nation, 27 de marzo de 1965, p. 2.

The Daily Nation. 1963. "Pledge may End Radio War". 13 de noviembre de 1963, núm. 968.

The Ethiopian Herald. 1978. "Reactionary War to Restore Forces". 12 de febrero de 1978, p. 2.

The New York Times. 1964a. "Ethiopian Planes destroy Two Somali Border Posts". 17 de enero de 1964, p. 8.

The New York Times. 1964b. "Somali Attack Reported". 13 de febrero de 1964, p. 2.

The New York Times. 1964c. "Ethiopia Charges Truce is Broken; Says Somali Troops Attack Three Points at Frontier". 17 de febrero de 1964, p. 1.

The Nerw York Times. 1964d. "Somali Border Fight Renewed". 7 de marzo de 1964, p. 12.

The New York Times. 1964f. "Ethiopian Force quits Border". 9 de abril de 1964, p. 17.

Torres García, Ana. 2010. "Historia de las relaciones exteriores del Marruecos independiente. La Guerra de las Arenas (1963) y la diplomacia occidental". Tesis de doctorado. Universidad de Sevilla. https://idus.us.es/xmlui/handle/11441/70361 
Turton, E. R. 1972. "Somali Resistance to Colonial Rule and the Development of Somali Political Activity in Kenya 18931960". Journal of African History 13(1): 119-143. https://doi. org/10.1017/S0021853700000293

U.S. Department of State Office of the Historian. 1999a. "290. Circular Airgram from the Department of State to Certain African Posts", 21 de marzo de 1964. Foreign Relations of the United States, 1964-1968. Vol. XXIV, Africa, editado por Nina Davis Howland. Washington: United States Government Printing Office. https://history.state.gov/historicaldocuments/frus1964$68 \mathrm{v} 24 / \mathrm{d} 290$

U.S. Department of State Office of the Historian. 1999b. "340. Telegram from the Embassy in Somalia to the Department of the State", 27 de julio de 1967. Foreign Relations of the United States, 1964-1968. Vol. XXIV, Africa, editado por Nina Davis Howland. Washington: United States Government Printing Office. https://history.state.gov/historicaldocuments/frus1964$68 \mathrm{v} 24 / \mathrm{d} 340 \#$ fn:1.5.4.2.36.208.12.2

WhitTAKer, Hannah. 2015. Insurgency and Counterinsurgency in Kenya: A Social History of the Shifta Conflict, c. 1963-1968. Leiden: Brill.

YAGY, Vatanyar Saidovich. 1990. "Ethiopia and its Neighbors: An Evolution of Relations, 1974-1989". Northeast African Studies 12(2/3): 107-116. http://www.jstor.org/stable/43660317

Pablo Arconada-Ledesma es investigador en la Universidad de Valladolid y miembro del Observatorio de Estudios Africanos. Es licenciado en historia y máster en relaciones internacionales y estudios africanos. Además, pertenece al Grupo de Investigación Reconocido Memoria Histórica, Derechos $\mathrm{Hu}$ manos y Transiciones Políticas. Cuenta con varios artículos publicados en revistas científicas nacionales e internacionales, así como diversos capítulos de libro. También es coordinador (junto con César García Andrés y Jara Cuadrado Bolaños) del volumen: África, un continente en transformación. Enfoques interdisciplinares (2020). Colabora con diferentes asociaciones del ámbito africano como Umoya, Nanouki y Wiriko. Ha 
ARCONADA: LA MEDIACIÓN DE LA ORGANIZACIÓN DE LA UNIDAD... 515 publicado en medios de comunicación como El País/Planeta Futuro, La Jornada, El Diario.es o Africaye.

http://orcid.org/0000-0003-4068-274X pablo.arconada@uva.es 
